\title{
Effect of High-Flow Nasal Cannula on Thoraco-Abdominal Synchrony in Adult Critically Ill Patients
}

\author{
Taiga Itagaki MD, Nao Okuda MD, Yumiko Tsunano MD, Hisakazu Kohata MD, \\ Emiko Nakataki MD PhD, Mutsuo Onodera MD, Hideaki Imanaka MD PhD, \\ and Masaji Nishimura MD PhD
}

\begin{abstract}
BACKGROUND: High-flow nasal cannula (HFNC) creates positive oropharyngeal airway pressure and improves oxygenation. It remains unclear, however, whether HFNC improves thoracoabdominal synchrony in patients with mild to moderate respiratory failure. Using respiratory inductive plethysmography, we investigated the effects of HFNC on thoraco-abdominal synchrony. METHODS: We studied 40 adult subjects requiring oxygen therapy in the ICU. Low-flow oxygen (up to $8 \mathrm{~L} / \mathrm{min}$ ) was administered via oronasal mask for $30 \mathrm{~min}$, followed by $\mathrm{HFNC}$ at $30-50 \mathrm{~L} / \mathrm{min}$. Respiratory inductive plethysmography transducer bands were circumferentially placed: one around the rib cage, and one around the abdomen. We measured the movement of the rib-cage and abdomen, and used the sum signal to represent tidal volume $\left(\mathrm{V}_{\mathrm{T}}\right)$ during mask breathing, and at 30 min during HFNC. We calculated the ratio of maximum compartmental amplitude (MCA) to $V_{T}$, and the phase angle. We assessed arterial blood gas and vital signs at each period, and mouth status during HFNC. We used multiple regression analysis to identify factors associated with improvement in thoraco-abdominal synchrony. RESULTS: During HFNC, breathing frequency significantly decreased from 25 breaths/min (IQR 22-27 breaths/min) to 21 breaths/min (IQR 1824 breaths $/$ min $)(P<.001)$, and $M C A / V_{\mathrm{T}}(P<.001)$ and phase angle $(P=.047)$ significantly improved. CONCLUSIONS: HFNC improved thoraco-abdominal synchrony in adult subjects with mild to moderate respiratory failure. Key words: high-flow oxygen therapy; nasal cannula; thoracoabdominal synchrony; respiratory inductive plethysmography; acute respiratory failure; critical care. [Respir Care 2014;59(1):70-74. () 2014 Daedalus Enterprises]
\end{abstract}

\section{Introduction}

Oronasal mask oxygen therapy and noninvasive ventilation are widely used in critical care settings. Noninvasive

\footnotetext{
The authors are affiliated with the Department of Emergency and Critical Care Medicine, University of Tokushima Graduate School, with the exception of Dr Imanaka, who is affiliated with the Department of Emergency and Disaster Medicine, Tokushima University Hospital, Tokushima, Japan.
}

The authors have disclosed no conflicts of interest.

Correspondence: Masaji Nishimura MD PhD, Department of Emergency and Critical Care Medicine, University of Tokushima Graduate School, 3-18-15 Kuramoto-cho, Tokushima, Japan 770-8503. E-mail address: nmasaji@tokushima-u.ac.jp.

DOI: $10.4187 /$ respcare. 02480 ventilation is often poorly tolerated, due to the close fitting of the mask, which hinders speech and oral intake., ${ }^{1,2}$ While nasal cannulae are less obstructive and allow speaking and drinking, ${ }^{3}$ conventional nasal cannulae cannot be used with high flow, which would cause nasal soreness or bleeding. ${ }^{4}$

High-flow nasal cannula (HFNC) can deliver adequately heated and humidified oxygen, at a maximum flow of $60 \mathrm{~L} / \mathrm{min}^{5-7}$ and HFNC has become a routine treatment in ICUs for spontaneously breathing patients with respiratory failure. $5,6,8,9$ The beneficial effects of HFNC have been widely reported: heated humidification during HFNC improves patient comfort ${ }^{8}$; flushing of anatomical dead space reduces $\mathrm{CO}_{2}$ level ${ }^{5,10}$; and the expiratory resistance produced by the continuous high flow causes a low level of positive oropharyngeal airway pressure..$^{5,11-13}$

In patients with respiratory failure, coordinated movement of the rib cage and abdominal wall is often impaired. 
Lack of coordination increases the work of breathing and causes respiratory muscle fatigue. ${ }^{14,15}$ Patients displaying asynchronous thoraco-abdominal movement have increased risk of ventilatory failure, necessitating mechanical ventilation and, consequently, poorer prognosis. ${ }^{16,17}$ To our knowledge, studies on thoraco-abdominal movement during HFNC are limited. In premature neonates, Saslow and colleagues compared phase angle during HFNC versus during nasal CPAP. ${ }^{18}$ In adults, HFNC was found to improve thoraco-abdominal synchrony, but was not quantitatively evaluated. ${ }^{19}$ The primary aim of the present study was to clarify the effects of HFNC on thoraco-abdominal synchrony in adult ICU patients with mild to moderate respiratory failure.

\section{Methods}

We conducted a prospective comparative study in a university hospital ICU. The study protocol was approved by the human ethics committee of Tokushima University Hospital, and informed consent was obtained from each subject's next of kin prior to inclusion.

\section{Study Population}

We studied adult patients with mild to moderate respiratory failure requiring oxygen therapy. The inclusion criteria were: $\mathrm{P}_{\mathrm{aO}_{2}} / \mathrm{F}_{\mathrm{IO}_{2}}<300 \mathrm{~mm} \mathrm{Hg}$; breathing frequency $>25$ breaths/min; dyspnea or accessory muscle use; asynchronous or paradoxical breathing pattern; and post-thoracotomy. The exclusion criteria were: mean blood pressure $<50 \mathrm{~mm} \mathrm{Hg} ; \mathrm{P}_{\mathrm{aO}_{2}} / \mathrm{F}_{\mathrm{IO}_{2}}<150 \mathrm{~mm} \mathrm{Hg}$; facial trauma or nasal obstruction; inability to tolerate HFNC; and Glasgow coma score $<12$.

\section{Study Protocol}

We recorded demographic data, Glasgow coma score, and Acute Physiology and Chronic Health Evaluation II score. Thoraco-abdominal movements were measured with respiratory inductive plethysmography (Respitrace, Ambulatory Monitoring, Ardsley, New York). Two elastic transducers (Respibands), which recorded changes in cross-sectional area, were attached to the body just prior to oronasal mask oxygen therapy. One band was placed around the rib cage, with the upper edge at the level of the axilla, and the other around the abdomen, with the upper edge at the level of the umbilicus. First, oxygen was administered via oronasal mask, at 3-8 L/min, for $30 \mathrm{~min}$, and then we measured thoraco-abdominal movement, heart rate, blood pressure, breathing frequency, and $\mathrm{S}_{\mathrm{pO}_{2}}$, and drew arterial blood samples. Next, oxygen was administered via HFNC (Optiflow HFNC, MR850 humidifier, RT319 heated delivery tube, and OPT544 nasal cannula, all from Fisher \&

\section{QUICK LOOK}

\section{Current knowledge}

High-flow nasal cannula (HFNC) creates positive oropharyngeal airway pressure, washes out the nasopharyngeal dead space, and improves oxygenation. The impact of HFNC on thoraco-abdominal synchrony has not been described.

\section{What this paper contributes to our knowledge}

HFNC improved thoraco-abdominal synchrony, by improving the phase angle and the ratio of maximum compartmental amplitude to tidal volume. HFNC also significantly reduced breathing frequency in patients with moderate hypoxemic respiratory failure.

Paykel Healthcare, Auckland, New Zealand), at $30 \mathrm{~L} / \mathrm{min}$. Flow was titrated according to subject tolerance. At 30 min after the start of HFNC, we acquired the same data as after mask oxygen. During HFNC we monitored whether the subject's mouth was open or closed, and rated it on a 3-point scale: poor $=$ almost always open, fair $=$ open about half the time, good $=$ almost always closed. HFNC was continued until, according to the judgment of attending physicians, the subject did not need oxygen.

We calculated $\mathrm{P}_{\mathrm{aO}_{2}} / \mathrm{F}_{\mathrm{IO}_{2}}$ during oronasal mask oxygen with $\mathrm{F}_{\mathrm{IO}_{2}}$ values calculated according to oxygen flow: $3 \mathrm{~L} /$ $\min =\mathrm{F}_{\mathrm{IO}_{2}} 0.3,5 \mathrm{~L} / \mathrm{min}=\mathrm{F}_{\mathrm{IO}_{2}} 0.4,7 \mathrm{~L} / \mathrm{min}=\mathrm{F}_{\mathrm{IO}_{2}} 0.5 .{ }^{20}$

\section{Measurement of Thoraco-Abdominal Movement}

Respiratory inductive plethysmography was conducted as follows. The motions of the rib cage and abdomen were measured as the changes in the inductance of the Respibands during oronasal mask and HFNC. We calculated the maximum compartmental amplitudes (MCAs) as the sums of the maximal peak-to-trough amplitudes of rib-cage and abdomen excursions. We then calculated the ratio of MCA to tidal volume $\left(\mathrm{V}_{\mathrm{T}}\right)$ with the maximal amplitude of the sum signal of rib cage and abdomen to resemble $\mathrm{V}_{\mathrm{T}}$. We created Konno-Mead diagrams by plotting abdomen motion against rib-cage motion. Phase angle $(\theta)$ was calculated according to the formula $\theta=$ $\sin ^{-1}(M / S)$, where $M$ is the horizontal distance on the Konno-Mead diagram at halfway between the maximal and minimal rib-cage excursion, and $\mathrm{S}$ is the maximal abdomen excursion (Figure).

\section{Statistical Analysis}

Data from before and after HFNC were compared with the Wilcoxon signed-rank test. To discover independent 

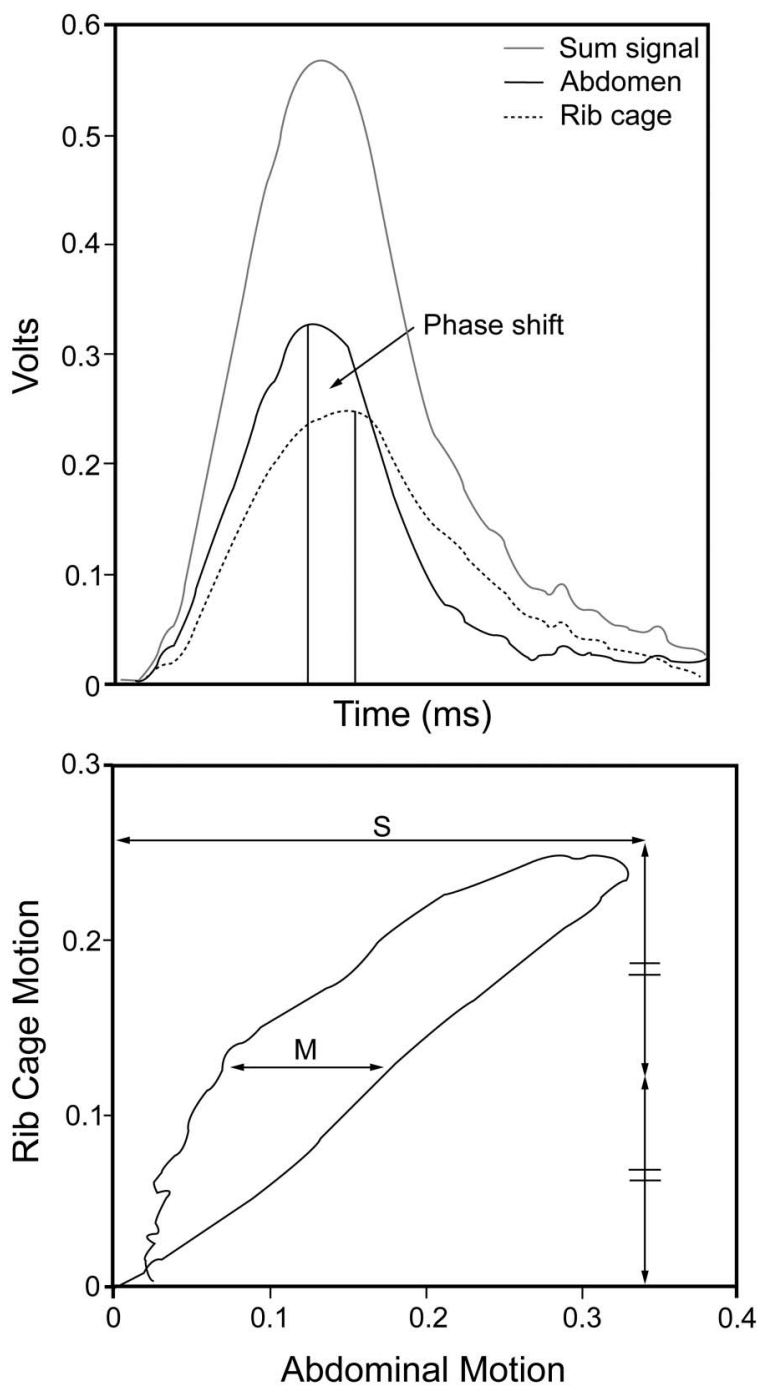

Figure. Two quantitative indexes of thoraco-abdominal synchrony, measured via respiratory inductive plethysmography. A: Rib-cage motion, abdominal motion, and their sum signal, which represents the tidal volume $\left(\mathrm{V}_{\mathrm{T}}\right)$. The maximum compartmental amplitude (MCA) is calculated as maximum abdominal motion + maximum rib-cage motion. $M C A / V_{T}$ approaches 1.0 with improving synchrony. B: Konno-Mead diagram of abdominal motion versus ribcage motion. The phase angle $(\theta)$ is calculated according to the formula $\theta=\sin ^{-1}(M / S)$, where $M$ is the horizontal distance of the Konno-Mead diagram at halfway between the maximal and minimal rib-cage excursion, and $S$ is the maximal abdominal excursion. Asynchrony produces a wide open loop. Synchronous ribcage/abdominal motion has a phase angle of $0^{\circ}$, and paradoxical motion has a phase angle of $180^{\circ}$.

factors that improved thoraco-abdominal synchrony, we used univariate and stepwise multiple regression analysis. Changes in $\mathrm{MCA} / \mathrm{V}_{\mathrm{T}}$ and phase angle were used as dependent variables. The continuous variables were subject age, body mass index (BMI), $\mathrm{P}_{\mathrm{aO}_{2}} / \mathrm{F}_{\mathrm{IO}_{2}}$ during oronasal mask, gas flow during HFNC, and difference in breathing frequency between oronasal mask and HFNC. We created
Table 1. Subjects $(n=40)$

\begin{tabular}{|c|c|}
\hline Age, median (IQR) y & $72(63-76)$ \\
\hline Male/female, no. & $25 / 15$ \\
\hline Height, median (IQR) $\mathrm{cm}$ & $162(151-168)$ \\
\hline Weight, median (IQR) kg & $58(49-67)$ \\
\hline Body mass index, median (IQR) & $23(20-25)$ \\
\hline APACHE II score, median (IQR) & $20(17-23)$ \\
\hline \multicolumn{2}{|l|}{ Care category, no. } \\
\hline Surgical & 27 \\
\hline Cardiovascular & 8 \\
\hline Thoracic & 5 \\
\hline Upper abdominal & 2 \\
\hline Lower abdominal & 5 \\
\hline Brain & 5 \\
\hline Orthopedic & 2 \\
\hline Medical & 13 \\
\hline Heart failure & 6 \\
\hline Sepsis & 2 \\
\hline COPD & 4 \\
\hline Neuromuscular disease & 1 \\
\hline \multicolumn{2}{|l|}{ Cause of respiratory failure, no. } \\
\hline Hypoxia $\left(\mathrm{P}_{\mathrm{aO}_{2}} / \mathrm{F}_{\mathrm{IO}_{2}}<300 \mathrm{~mm} \mathrm{Hg}\right)$ & 19 \\
\hline Tachypnea $(>25$ breaths/min $)$ & 9 \\
\hline Dyspnea/forced breathing & 10 \\
\hline $\begin{array}{l}\text { Abnormal (asynchronous or paradoxical) } \\
\text { breathing pattern }\end{array}$ & 2 \\
\hline $\begin{array}{l}\text { Consciousness disturbance (Glasgow coma } \\
\text { score 12-14) }\end{array}$ & 16 \\
\hline Post-thoracotomy & 9 \\
\hline
\end{tabular}

dummy variables with the values 1 and 0 for the nominal variables sex $(1=$ male, $0=$ female $)$, consciousness disturbance $(1=$ Glasgow coma score of $15,0=$ Glasgow coma score of 12-14), and mouth open/closed status during $\operatorname{HFNC}(1=$ good or fair, $0=$ poor $)$. In the univariate analysis we considered $P<.01$ significant (ie, variables possibly associated with the effect of HFNC), and then assessed those variables in stepwise multiple regression analysis. We considered as possible confounding factors the gas flow and mouth open/closed status during HFNC, and the difference in breathing frequency between mask and HFNC. Statistical calculations were carried out with statistics software (SPSS 11.0.1, SPSS, Chicago, Illinois). Data are expressed as median and interquartile range. $P<.05$ was considered statistically significant.

\section{Results}

Fifty patients were eligible for inclusion, but consent was not obtained from 3 patients, and 7 patients were too restless to record thoraco-abdominal movement, so 40 patients were included (Table 1). There were more males 
Effect of High-Flow Nasal Cannula on Thoraco-Abdominal Synchrony

Table 2. Ventilation and Physiologic Variables During Oronasal Mask Oxygen Therapy Versus High-Flow Nasal Cannula

\begin{tabular}{|c|c|c|c|}
\hline & Oronasal Mask & High-Flow Nasal Cannula & $P$ \\
\hline Flow, L/min & $5(5-5)$ & $40(35-40)$ & $<.001$ \\
\hline $\mathrm{F}_{\mathrm{IO}_{2}}$ & $0.39(0.40-0.40)$ & $0.36(0.34-0.40)$ & .02 \\
\hline Breathing frequency, breaths/min & $25(22-27)$ & $21(18-24)$ & $<.001$ \\
\hline $\mathrm{pH}$ & $7.46(7.41-7.50)$ & $7.46(7.41-7.50)$ & .06 \\
\hline $\mathrm{P}_{\mathrm{aO}_{2}}, \mathrm{~mm} \mathrm{Hg}$ & $97(78-130)$ & $101(77-116)$ & .75 \\
\hline $\mathrm{P}_{\mathrm{aCO}}, \mathrm{mm} \mathrm{Hg}$ & $36(33-40)$ & $36(34-40)$ & .22 \\
\hline Mean blood pressure, $\mathrm{mm} \mathrm{Hg}$ & $88(77-101)$ & $87(73-97)$ & .21 \\
\hline Heart rate, beats/min & $89(78-104)$ & $91(79-102)$ & .32 \\
\hline Mouth closure: poor/fair/good, no. & NA & $17 / 12 / 11$ & NA \\
\hline $\mathrm{MCA} / \mathrm{V}_{\mathrm{T}}$ & $1.02(1.01-1.05)$ & $1.00(1.00-1.02)$ & $<.001$ \\
\hline Phase angle, ${ }^{\circ}$ & $19.3(11.0-26.8)$ & $12.6(6.4-25.9)$ & .047 \\
\hline \multicolumn{4}{|c|}{$\begin{array}{l}\text { Values are median and IQR unless otherwise indicated. } \\
\text { NA }=\text { not applicable }\end{array}$} \\
\hline
\end{tabular}

than females and more surgical subjects, half of whom underwent cardiothoracic procedures. The most frequent cause of respiratory failure was hypoxia (19 subjects).

There were no statistically significant differences between mask and $\mathrm{HFNC}$ in $\mathrm{pH}, \mathrm{P}_{\mathrm{aO}_{2}}, \mathrm{P}_{\mathrm{aCO}}$, mean blood pressure, and heart rate (Table 2). HFNC significantly decreased breathing frequency $(P<.001)$ and significantly improved MCA/ $\mathrm{V}_{\mathrm{T}}(P<.001)$ and phase angle $(P=.047)$.

Results of stepwise multiple regression analysis showed that no items were associated with improved $M C A / V_{T}$. Likewise, no items independently affected the improvement of phase angle.

\section{Discussion}

HFNC improved thoraco-abdominal synchrony, as shown by statistically significant improvement of MCA/ $\mathrm{V}_{\mathrm{T}}$ and phase angle, and decreased the breathing frequency. This is the first quantitative investigation of the shortterm effects of HFNC on thoraco-abdominal synchrony in adult subjects with mild to moderate respiratory failure. While Sztrymf and colleagues reported how HFNC affects thoraco-abdominal synchrony, they did not quantitatively evaluate the effects. ${ }^{19}$ Using $\mathrm{MCA} / \mathrm{V}_{\mathrm{T}}$ and phase angle, which are superior to other indices for quantitatively assessing breathing pattern, ${ }^{21,22}$ we evaluated thoracoabdominal synchrony.

When a patient is not mandated to keep the mouth closed, HFNC delivers a low level of positive airway pressure $\left(<3 \mathrm{~cm} \mathrm{H}_{2} \mathrm{O}\right)^{5,11-13,23}$ It is not fully understood, however, whether or not the benefits of HFNC are induced solely by positive airway pressure. ${ }^{8}$ Though HFNC increases positive airway pressure, ${ }^{5,9,11,24}$ we found no correlation of breathing-pattern improvement with female sex, mouth closure, or high gas flow. These findings suggest that positive airway pressure is not the only HFNC mechanism contributing to improved breathing pattern. It is likely that enhanced patient comfort from heated humidification, ${ }^{8}$ flushing of anatomical dead space, and reduction of airway resistance ${ }^{5,10}$ lessen the work of breathing and thus improve thoraco-abdominal synchrony.

Corley and colleagues reported that, as BMI increases, so does end-expiratory lung volume with HFNC. They speculated that obese patients might derive benefit more from HFNC because they have more recruitable alveoli. ${ }^{25}$ Our subjects' BMIs were all within the normal range, compared to the study by Corley and colleagues, which may explain why we found no correlation between $\mathrm{MCA} / \mathrm{V}_{\mathrm{T}}$ improvement and BMI. Further study with oropharyngeal airway pressure measurements may reveal how positive airway pressure affects the respiratory mechanics of patients with different body sizes.

The statistically significant short-term decrease in breathing frequency in our study is consistent with previous studies. ${ }^{7,19,25,26}$ While improved thoraco-abdominal synchrony might be a mechanism of breathing-frequency reduction, we found no significant reduction in $\mathrm{P}_{\mathrm{aCO}}$. Roca and colleagues similarly reported that HFNC significantly reduced breathing frequency, but with no reduction in $\mathrm{P}_{\mathrm{aCO}_{2}}{ }^{7}$ We did not perform quantitative calibrations for respiratory inductive plethysmography, so we were unable to obtain accurate $V_{T}$ from each subject with respiratory inductive plethysmography. Even so, the product of the sum signal and breathing frequency was statistically significantly reduced by HFNC $(P<.001)$. If minute volume was reduced by HFNC without any change in alveolar ventilation, it is likely that HFNC reduced anatomical dead space. 


\section{Limitations}

First, this was not a controlled trial, and we did not conduct the 2 oxygen therapies at random. A randomized crossover trial with sufficiently long wash-out periods would reveal changes with HFNC and clarify HFNC's effect on thoraco-abdominal synchrony. Second, it is uncertain whether $30 \mathrm{~min}$ for each oxygen therapy was adequate, but a long study duration would make it difficult to clarify the effects of HFNC versus the effects of other treatments (eg, antibiotics, bronchodilators). Third, beginning with a higher flow might have given different results. Fourth, we studied only 40 mixed medical and surgical subjects, and only subjects with mild to moderate respiratory failure, so we cannot extrapolate our findings directly to other types of patients. With more subjects and more types of subjects, multivariate analysis might have revealed HFNC benefits in specific types of patients. Finally, we were unable to elucidate the mechanisms by which HFNC improved thoraco-abdominal synchrony. Measurements of oropharyngeal airway pressure and transpulmonary pressure (derived from esophageal pressure) might help clarify this question.

\section{Conclusions}

HFNC immediately improved thoraco-abdominal synchrony, as demonstrated by reduced $\mathrm{MCA} / \mathrm{V}_{\mathrm{T}}$, phase angle, and breathing frequency in adult subjects with mild to moderate respiratory failure.

\section{REFERENCES}

1. Garpestad E, Brennan J, Hill NS. Noninvasive ventilation for critical care. Chest 2007;132(2):711-720.

2. Nava S, Ceriana P. Causes of failure of noninvasive mechanical ventilation. Respir Care 2004;49(3):295-303.

3. Tiruvoipati R, Lewis D, Haji K, Botha J. High-flow nasal oxygen vs high-flow face mask: a randomized crossover trial in extubated patients. J Crit Care 2010;25(3):463-468.

4. American Association for Respiratory Care. aarc clinical practice guideline. Oxygen therapy for adults in the acute care facility: 2002 revision \& update. Respir Care 2002;47(6):717-720.

5. Lee JH, Rehder KJ, Williford L, Cheifetz IM, Turner DA. Use of high flow nasal cannula in critically ill infants, children, and adults: a critical review of the literature. Intensive Care Med 2013;39(2): 247-257.

6. Parke RL, McGuinness SP, Eccleston ML. A preliminary randomized controlled trial to assess effectiveness of nasal high-flow oxygen in intensive care patients. Respir Care 2011;56(3):265-270.

7. Roca O, Riera J, Torres F, Masclans JR. High-flow oxygen therapy in acute respiratory failure. Respir Care 2010;55(4):408-413.

8. Dysart K, Miller TL, Wolfson MR, Shaffer TH. Research in high flow therapy: mechanisms of action. Respir Med 2009;103(10): 1400-1405.
9. Kernick J, Magarey J. What is the evidence for the use of high flow nasal cannula oxygen in adult patients admitted to critical care units? A systematic review. Aust Crit Care 2010;23(2):53-70.

10. Dewan NA, Bell CW. Effect of low flow and high flow oxygen delivery on exercise tolerance and sensation of dyspnea. A study comparing the transtracheal catheter and nasal prongs. Chest 1994; 105(4):1061-1065.

11. Groves N, Tobin A. High flow nasal oxygen generates positive airway pressure in adult volunteers. Aust Crit Care 2007;20(4):126-131.

12. Parke RL, Eccleston ML, McGuinness SP. The effects of flow on airway pressure during nasal high-flow oxygen therapy. Respir Care 2011;56(8):1151-1155.

13. Parke R, McGuinness S, Eccleston M. Nasal high-flow therapy delivers low level positive airway pressure. Br J Anaesth 2009;103(6): 886-890.

14. Clergue F, Whitelaw WA, Charles JC, Gandjbakhch I, Pansard JL, Derenne JP, Viars P. Inferences about respiratory muscle use after cardiac surgery from compartmental volume and pressure measurements. Anesthesiology 1995;82(6):1318-1327.

15. Tobin MJ, Jenouri G, Lind B, Watson H, Schneider A, Sackner MA. Validation of respiratory inductive plethysmography in patients with pulmonary disease. Chest 1983;83(4):615-620.

16. Ashutosh K, Gilbert R, Auchincloss JH, Peppi D. Asynchronous breathing movements in patients with chronic obstructive pulmonary disease. Chest 1975;67(5):553-557.

17. Gilbert R, Ashutosh K, Auchincloss JH, Rana S, Peppi D. Prospective study of controlled oxygen therapy. Poor prognosis of patients with asynchronous breathing. Chest 1977;71(4):456-462.

18. Saslow JG, Aghai ZH, Nakhla TA, Hart JJ, Lawrysh R, Stahl GE, Pyon KH. Work of breathing using high-flow nasal cannula in preterm infants. J Perinatol 2006;26(8):476-480.

19. Sztrymf B, Messika J, Bertrand F, Hurel D, Lwon R, Dreyfuss D, Ricard JD. Beneficial effects of humidified high flow nasal oxygen in critical care patients: a prospective pilot study. Intensive Care Med 2011;37(11):1780-1786.

20. Branson RD, Hess DR, Chatburn RL. Respiratory care equipment, 2nd edition. New York: Lippincott Williams \& Wilkins; 1999:66-67.

21. Beydon N, Davis SD, Lombardi E, Allen JL, Arets HG, Aurora P, et al. An official American Thoracic Society/European Respiratory Society statement: pulmonary function testing in preschool children. Am J Respir Crit Care Med 2007;175(12):1304-1345.

22. Tobin MJ. Breathing pattern analysis. Intensive Care Med 1992; 18(4):193-201.

23. Urbano J, del Castillo J, López-Herce J, Gallardo JA, Solana MJ, Carrillo Á. High-flow oxygen therapy: pressure analysis in a pediatric airway model. Respir Care 2012;57(5):721-726.

24. Frizzola M, Miller TL, Rodriguez ME, Zhu Y, Rojas J, Hesek A, et al. High-flow nasal cannula: impact on oxygenation and ventilation in an acute lung injury model. Pediatr Pulmonol 2011;46(1): 67-74.

25. Corley A, Caruana LR, Barnett AG, Tronstad O, Fraser JF. Oxygen delivery through high-flow nasal cannulae increase end-expiratory lung volume and reduce respiratory rate in post-cardiac surgical patients. Br J Anaesth 2011;107(6):998-1004.

26. Sztrymf B, Messika J, Mayot T, Lenglet H, Dreyfuss D, Ricard JD. Impact of high-flow nasal cannula oxygen therapy on intensive care unit patients with acute respiratory failure: a prospective observational study. J Crit Care 2012;27(3):324.e9-e13. 